\title{
The Impact of Coating Ingredients on the Aging Resistance of Topcoat Paints by Model Trees
}

\author{
Tzu-Tsung Wong ${ }^{*}$, Shih-Hsuan Hung \\ Institute of Information Management, National Cheng Kung University, Tainan, Taiwan \\ Received 20 May 20xx; received in revised form 15 July 2020; accepted 14 September 2020 \\ DOI: https://doi.org/10.46604/aiti.2021.5307
}

\begin{abstract}
Topcoat paint is mainly composed of resin and pigment and hence its quality highly depends on the type and proportion of these two ingredients. This study aims at testing the formula of the topcoat paint for finding one that can achieve better quality for anti-aging. Various formulas of paint are applied on boards that will be put into ultraviolet accelerated test machines to simulate weathering tests. The gloss and color, before and after the tests, are collected and numerical prediction method M5P is used to grow model trees for discovering the key factors affecting aging. Based on the structure and the linear regression models in the trees, a better topcoat paint should be composed of a high proportion of resin and generally a low proportion of pigment. Good types of resin and pigment are also identified for keeping color and gloss.
\end{abstract}

Keywords: accelerated aging test, linear regression, model tree, numeric prediction, topcoat paint

\section{Introduction}

Buildings are usually covered by paint to enhance their looking and durability. There are two main categories of paint: undercoating and topcoating. Topcoat paint can protect buildings from erosion and the function of undercoat paint is to increase the adhesion of topcoat paint. The life of a building is thus primarily determined by the quality of topcoat paint. The two main materials for composing paint are resin and pigment. The type and proportion of these two ingredients have a great impact on the appearance and aging-resistance properties of topcoat paint. If the relationship between the ingredients and the aging properties can be determined, an enterprise will be able to produce a topcoat paint that can satisfy the requirements of a customer at a relatively low cost.

The coating on objects is critical to the protection of the objects and hence this kind of the issue has been studied in several applications [1-4]. Accelerated tests were generally adopted to obtain observations for studying the properties of paints [5-7]. Resin and pigment are the two main ingredients of paints. Many recent studies attempted to explore the individual impact of resin [8-9] or pigment [10-14]. These two ingredients can also be considered together to determine the performance of coatings [15-17]. None of those previous studies applied numeric prediction methods to analyse the impact of resin and pigment on the aging properties of paints.

The purpose of this paper is to explore the various formulas of topcoat paint. The data collected for various formulas will be analysed by a numeric prediction method that can produce a tree structure with multiple linear regression models. The tree and the regression models can be helpful in finding the attributes that are critical to the properties of topcoat paint. These results can be employed to compose the topcoat paint for satisfying customer needs.

* Corresponding author. E-mail address: tzutsung@ mail.ncku.edu.tw

Tel.: +886-6-2757575; Fax: +886-6-2362162 


\section{Data Collection}

The three factors that can affect the life of paint are solar radiation, temperature, and humidity, and solar radiation has the most significant impact. Better quality of resin and pigment can enhance the aging-resistant properties of paint. A company uses three types of resin $r_{1}, r_{2}$, and $r_{3}$ and three types of pigment $p_{1}, p_{2}$, and $p_{3}$ provided by different vendors to produce paint. The proportion of the resin in a formula can be between $30 \%$ and $60 \%$, and this value for pigment is generally between $15 \%$ and $35 \%$. The four attributes for determining the aging-resistant properties of paint are shown in Table 1 . The number of possible combinations resulting from the four attributes is $3 \times 4 \times 3 \times 5=180$. The paint of each combination will be applied on two boards to collect their data for the aging resistance; accordingly, the number of instances in a data set will be 360 . Note that the two proportions $X_{2}$ and $X_{4}$ are considered as continuous attributes for analysing their impact on the age-resistant properties.

Table 1 The attributes for determining the aging-resistant properties of paint

\begin{tabular}{|c|c|}
\hline Attribute & Possible values \\
\hline$X_{1}$ (Resin type) & $r_{1}, r_{2}, r_{3}$ \\
\hline$X_{2}$ (Resin proportion) & $30 \%, 40 \%, 50 \%, 60 \%$ \\
\hline$X_{3}$ (Pigment type) & $p_{1}, p_{2}, p_{3}$ \\
\hline$X_{4}$ (Pigment proportion) & $15 \%, 20 \%, 25 \%, 30 \%, 35 \%$ \\
\hline
\end{tabular}

The lifetime of a system is the duration for the system to operate normally. However, the degradation of the coating in a building occurs gradually as time goes by. The age of paint is thus represented by its colour and gloss. An aluminium board coating by paint will be dried naturally for a week. Then its colour and gloss will be measured and recorded as $c_{0}$ and $g_{0}$, separately. Fig. 1 shows the colour meter to evaluate the colour in a board. For the sake of simplicity, the colour of all formulas is set to be white. A smaller value implies that the white colour is brighter. Similarly, the gloss of a board is measured by the gloss meter shown in Fig. 2. The gloss values of five fixed positions in a board are measured, and their average will be the gloss level of the board.

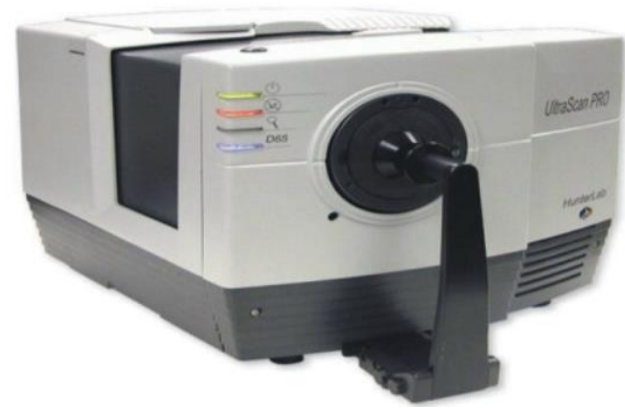

Fig. 1 The colour meter

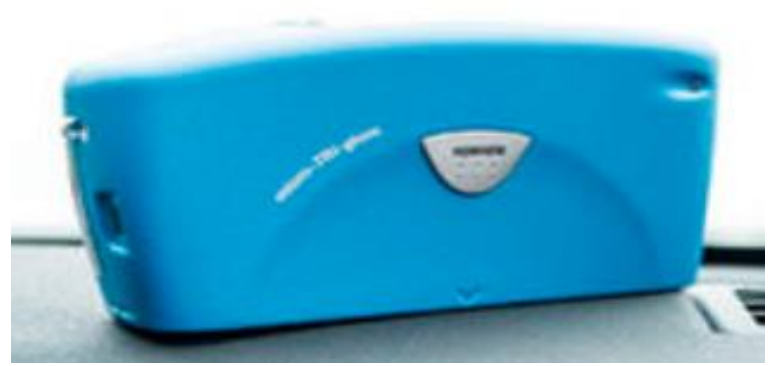

Fig. 2 The gloss meter

After the initial color $c_{0}$ and gloss $g_{0}$ of each board had been obtained, the 360 boards were put into ultraviolet accelerated test machines for $t$ hours. Then the color and gloss of each board were measured again as before, and they were denoted as $c_{1}$ and $g_{1}$, respectively. This testing process in the ultraviolet accelerated test machines lasted $5 t$ hours, and every board was taken out the machine for measuring color and gloss every $t$ hours. The exposure conditions of the machine were set to be the Cycle 2 of X2.1 given in ASTM G154-16 provided by ASTM International. Let $c_{j}$ and $g_{j}$ be the color and the gloss of a board at $j \times t$ hours for $j=1,2,3,4,5$. The color difference for $j \times t$ hours was calculated as $d_{j}=c_{j}-c_{0}$, and the gloss retention rate for $j \times t$ hours was computed as $e_{j}=g_{j} / g_{0}$. The data set for color difference collected at $j \times t$ hours was denoted as $D_{j}$ in which every instance was represented as $\left\langle x_{1}, x_{2}, x_{3}, x_{4}, d_{j}\right\rangle$. Similarly, the data set for gloss retention rate collected at $j \times t$ hours was denoted as $E_{j}$ in which every instance was represented as $\left\langle x_{1}, x_{2}, x_{3}, x_{4}, e_{j}\right\rangle$.

Let the paint coating in a board be the formula with $40 \%$ type $r_{1}$ resin and $30 \%$ type $p_{2}$ pigment. The color and gloss measured after one-week natural drying are $c_{0}=0.1$ and $g_{0}=60$, individually. After the board is put into an ultraviolet accelerated test machine for $2 \times t$ hours, its color and gloss becomes $c_{2}=0.5$ and $g_{2}=51$, separately. The instances for this board 
in data sets $D_{2}$ and $E_{2}$ are $\left\langle r_{1}, 0.4, p_{2}, 0.3,0.4\right\rangle$ and $\left\langle r_{1}, 0.4, p_{2}, 0.3,0.85\right\rangle$, respectively. It is possible that the aging-resistant properties of formula may degrade slowly at the beginning while its degrading becomes quick after a fixed time point or vice versa. The 10 data sets can provide this kind of information to satisfy the needs of topcoat paints.

\section{Model Trees and Data Analysis}

Linear regression is the most popular tool for numeric prediction. It provides a linear model to analyze the relationships between independent variables and a dependent variable. When the instances in a data set are not collected from the same population, the prediction error resulting from single linear regression model is generally large. More powerful tools for numeric prediction are therefore developed to make more accurate predictions [18-21]. The model tree proposed by Quinlan [19] has a tree structure and multiple linear regression models for numeric prediction and interpretation, and its prediction error is generally far smaller than that resulting from single linear regression model. This tool has been employed in several recent applications for numeric prediction [22-26]. This section will briefly describe the way for building a model tree from a data set. The evaluation and the interpretation of a model tree will also be introduced.

\subsection{Model trees}

It is similar to decision trees, model trees have a tree structure that contains internal nodes for branching and leaf nodes for prediction. Every leaf node in a model tree has a linear regression model to predict the class value of any instance reaching this node. The attribute chosen for branching is determined by its standard deviation reduction. The domain of a continuous attribute is divided into ten equal-width intervals by nine splitting points. The expected standard deviation of the class value for every binary branching on each of the nine splitting points is calculated and the one with the smallest expected standard deviation will be the best splitting point for this attribute. The one that has the smallest expected standard deviation on the class value among all attributes is chosen for branching.

After the tree is fully grown, a linear regression model is derived for each node based on the instances reaching that node. The regression models in internal nodes will be used for pruning and the regression models in leaf nodes are employed for predicting the class value. A discrete attribute with $m$ possible values is replaced by $m-1$ synthetic binary attributes. The expected standard deviation of the class value for every binary attribute can be calculated to determine whether this attribute should be chosen for binary branching in an internal node. Example 1 given below shows the way to interpret the learning results for discrete attributes.

Example 1: Suppose that a data set has one continuous attribute $X_{1}$ and one discrete attribute $X_{2}$ with four possible values $a, b, c$, and $d$ for predicting class value $Y$. The order of these values is $c, d, a, b$ based on their average class value summarized from the data set; i.e., values $c$ and $b$ have the largest and the smallest average class values, respectively. Then the three synthetic binary attributes for replacing $X_{2}$ are given in Table 2. If the attribute value of $X_{2}$ appears in a binary attribute, the value of this binary attribute equals 1 and equals zero otherwise.

For example, an instance $\langle 0.4, d\rangle$ will be replaced by $\langle 0.4,1,0,0\rangle$. Let the linear regression equation in the leaf node reaching by this instance be $y=1.26 x_{1}-0.35 d-a-b+0.082 b$. Then the predicted class value of this instance is $y=1.26 \times 0.4-$ $0.35 \times 1+0.082 \times 0=0.154$.

Table 2 The synthetic binary attributes for replacing the discrete attribute $X_{2}$ in Example 1

\begin{tabular}{|c|c|c|c|}
\hline Attribute value & $d-a-b$ & $a-b$ & $b$ \\
\hline$a$ & 1 & 1 & 0 \\
\hline$b$ & 1 & 1 & 1 \\
\hline$c$ & 0 & 0 & 0 \\
\hline$d$ & 1 & 0 & 0 \\
\hline
\end{tabular}




\subsection{Evaluation and interpretation}

A small prediction error is the primary concern for numeric prediction. Since the color difference and the gloss retention rate have different scales, an evaluation measure should be able to indicate whether a formula of topcoat paint can achieve a small prediction error on both aging-resistance properties. Relative absolute error (RAE) and root relative squared error (RRSE) are therefore adopted for performance evaluation. Let $y_{i}$ and $y_{i}$ be the actual and the predicted class value of instance $i$. Then these two measures are calculated as:

$$
\mathrm{RAE}=\frac{\Sigma_{i}\left|y_{i}^{\prime}-y_{i}\right|}{\Sigma_{i}\left|y_{i}-\bar{y}\right|}
$$

and

$$
\operatorname{RRSE}=\sqrt{\frac{\sum_{i}\left(y_{i}^{\prime}-y_{i}\right)^{2}}{\sum_{i}\left(y_{i}-\bar{y}\right)^{2}}}
$$

where $\bar{y}$ is the average class value. These two evaluation measures have been normalized, and hence they can be employed to compare the prediction error between the color difference and the gloss retention rate.

Like decision trees, the branching attributes in a model tree determine the leaf node in which an instance will reach. Moreover, the linear regression models in leaf nodes will show critical attributes and their impact level on the dependent variable. There are five model trees for the color difference and similarly for the gloss retention rate. The five model trees for each aging-resistant property will be put together to analyze the critical attributes and their impact levels. Those results can be helpful to know which kind of formula will be better for aging resistance.

\section{Experimental Results}

As described in Section 2, the color and gloss of each board were measured for every $t$ hours. The value of $t$ was set to be 250 hours; therefore, the data were collected at $0,250,500,750,1000$, and 1250 hours. The class values in the data sets $D_{1}$ through $D_{5}$ and $E_{1}$ through $E_{5}$ are collected by this way, and they are divide into two groups for the color difference and the gloss retention ratio. These two groups of data sets will be analyzed by the function M5P with default settings provided by the software Weka in the following two subsections for drawing conclusions. Only the analysis on the data sets $D_{2}$ and $E_{2}$ obtained at 500 hours will be shown in detail because their learning results are simpler for interpretation. Then the five data sets in each group will be put together for identifying the critical attributes of topcoat paint in anti-aging. Since estimating the lifetime of topcoat paint is not the goal of this study, setting $t=250$ hours ensures that enough data can be collected within reasonable time.

\subsection{Color difference}

Table 3 The characteristics of the five model trees for colour difference

\begin{tabular}{|c|c|c|c|c|c|}
\hline Data set & $D_{1}$ & $D_{2}$ & $D_{3}$ & $D_{4}$ & $D_{5}$ \\
\hline RAE & $81.7 \%$ & $76.2 \%$ & $75.2 \%$ & $74.2 \%$ & $73.9 \%$ \\
\hline RRSE & $86.1 \%$ & $84.8 \%$ & $80.8 \%$ & $80.6 \%$ & $79.3 \%$ \\
\hline Branching attributes & $X_{1}, X_{2}, X_{3}$ & $X_{1}, X_{2}, X_{3}$ & $X_{1}, X_{2}, X_{3}$ & $X_{1}, X_{2}, X_{3}$ & $X_{1}, X_{2}, X_{3}, X_{4}$ \\
\hline Tree size & 8 & 4 & 9 & 8 & 8 \\
\hline
\end{tabular}

The prediction error and the size of the five model trees for the color difference are summarized in Table 3 in which tree size represents the number of leaf nodes. Both the relative absolute error and the root relative square error are getting smaller as 
the number of testing hours is increasing. The prediction on color difference is more accurate on the topcoat paint exposing longer. Attribute $X_{4}$ appears only in the tree structure for data set $D_{5}$. This suggests that pigment proportion is the least important among the four attributes.

The model tree and its corresponding linear regression models for data set $D_{2}$ are shown in Fig. 3 and Table 4, separately. Attribute $X_{4}$ (pigment proportion) is the only one that is not chosen for branching; consequently, it is less important than the other three attributes in determining the color difference of topcoat paints. According to the four linear regression models, the overall coefficients for resin types $r_{1}, r_{2}$, and $r_{3}$ are $0,-0.0670+0.0950=0.0280$, and $-0.1665-0.0670+0.0184+0.0950+$ $0.0184+0.0310=-0.0697$, respectively. These imply that the best and the worst resin types for reducing color difference are $r_{3}$ and $r_{2}$, respectively. Since the four coefficients for resin proportion are all negative, higher resin proportion is better for reducing color difference. Similar to resin types, the overall coefficients for pigment types $p_{1}, p_{2}$, and $p_{3}$ are $0,0.0314$, and 0.4623 , respectively. The priority order for pigment types is thus $p_{1}, p_{2}$, and $p_{3}$. The three coefficients for pigment proportion are all positive; therefore, the pigment proportion should not be high for maintaining the color of topcoat paint.

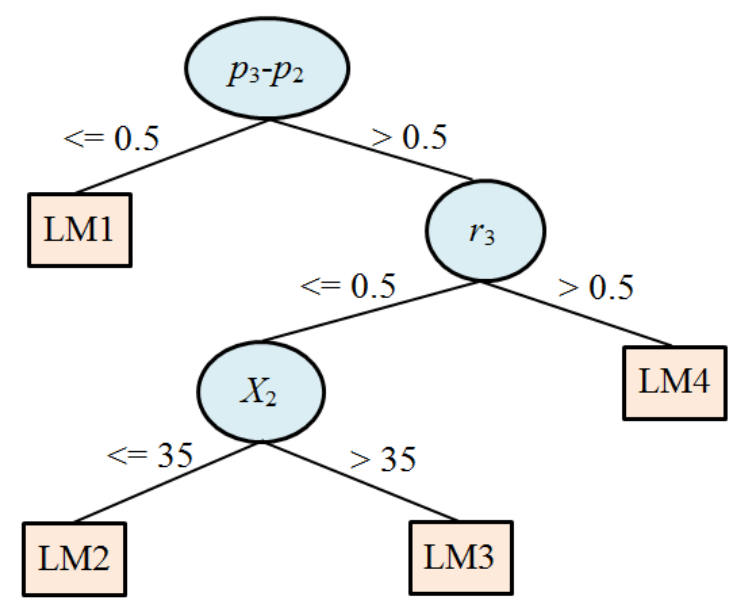

Fig. 3 The model tree grown from data set $D_{2}$

Table 4 The linear regression models for the model tree given in Fig. 3

\begin{tabular}{|c|c|c|c|c|}
\hline Attribute & LM1 & LM2 & LM3 & LM4 \\
\hline$r_{2}-r_{3}$ & & -0.0670 & 0.0950 & \\
\hline$r_{3}$ & -0.1655 & 0.0184 & 0.0184 & 0.0310 \\
\hline$X_{2}$ & -0.0032 & -0.0013 & -0.0008 & -0.0006 \\
\hline$p_{3}-p_{2}$ & 0.0122 & 0.0064 & 0.0064 & 0.0064 \\
\hline$p_{2}$ & 0.0116 & 0.2462 & -0.0472 & 0.2203 \\
\hline$X_{4}$ & & 0.0004 & 0.0004 & 0.0136 \\
\hline Constant & 0.7149 & 0.6290 & 0.5873 & 0.3376 \\
\hline
\end{tabular}

The above analysis is applied to all of the five model trees for the color difference and the results are summarized in Table 5. When the testing time is more than 500 hours, the best and the worst resin types are $r_{1}$ and $r_{2}$, respectively. Higher resin proportion is definitely beneficial for maintaining the color of topcoat paints. The ranks of pigment types are consistent all the time. Table 5 also suggests that the pigment proportion should not be high. According to the experimental results given in Table 5, the best formula for the color of topcoat paint is the combination of a high proportion of $r_{1}$ resin and a low proportion of $p_{1}$ pigment.

Table 5 The summarization of the linear regression models for colour difference

\begin{tabular}{cccccc}
\hline Data set & $D_{1}$ & $D_{2}$ & $D_{3}$ & $D_{4}$ & $D_{5}$ \\
\hline Rank of resin types & $r_{2}, r_{1}, r_{3}$ & $r_{1}, r_{3}, r_{2}$ & $r_{1}, r_{3}, r_{2}$ & $r_{1}, r_{3}, r_{2}$ & $r_{1}, r_{3}, r_{2}$ \\
Resin proportion & Negative & Negative & Negative & Negative & Negative \\
Rank of pigment types & $p_{1}, p_{3}, p_{2}$ & $p_{1}, p_{3}, p_{2}$ & $p_{1}, p_{3}, p_{2}$ & $p_{1}, p_{3}, p_{2}$ & $p_{1}, p_{3}, p_{2}$ \\
Pigment proportion & Negative & Positive & Positive & Positive & Positive \\
\hline
\end{tabular}




\subsection{Gloss retention ratio}

Table 6 shows the prediction errors and the sizes of the five model trees for the gloss retention rate. The testing time in the machines seems to have almost no impact on the prediction error. All five models trees have branching attributes resin type $\left(X_{1}\right)$; as a result, it is the most important attribute for gloss. Pigment type $\left(X_{3}\right)$ and pigment proportion $\left(X_{4}\right)$ become more important as exposing time is getting longer. Note that the prediction errors for the gloss retention rate are all smaller than those given in Table 3 for the color difference.

Table 6 The characteristics of the five model trees for gloss retention rate

\begin{tabular}{|c|c|c|c|c|c|}
\hline Data set & $E_{1}$ & $E_{2}$ & $E_{3}$ & $E_{4}$ & $E_{5}$ \\
\hline RAE & $65.3 \%$ & $59.5 \%$ & $68.3 \%$ & $65.1 \%$ & $65.4 \%$ \\
\hline RRSE & $68.9 \%$ & $61.5 \%$ & $70.9 \%$ & $67.5 \%$ & $67.7 \%$ \\
\hline Branching attributes & $X_{1}, X_{2}, X_{3}$ & $X_{1}, X_{2}, X_{4}$ & $X_{1}, X_{2}, X_{3}, X_{4}$ & $X_{1}, X_{3}, X_{4}$ & $X_{1}, X_{2}, X_{3}, X_{4}$ \\
\hline Tree size & 8 & 6 & 6 & 5 & 8 \\
\hline
\end{tabular}

The model tree and its corresponding linear regression models for data set $E_{2}$ are shown in Fig. 4 and Table 7, respectively. The only attribute that is not chosen for branching is $X_{3}$ (pigment type), and hence it is less important than the other three attributes in determining the gloss of topcoat paints. A small color difference is preferred, while better topcoat paint should have a larger gloss retention rate. The coefficients for resin types $r_{1}, r_{2}$, and $r_{3}$ calculated from linear regression model LM1 in Table 7 are $1.6714+0.7991=2.4705,1.6714$, and 0 , respectively. As a result, the best and the worst resin types for maintaining gloss are $r_{1}$ and $r_{3}$, respectively. The same result can be obtained from the other five linear regression models. The four coefficients for resin proportion are all positive; accordingly, higher resin proportion is better for the gloss retention rate. The coefficients for pigment types $p_{1}, p_{2}$, and $p_{3}$ calculated from LM1 are 21.9847-17.9402 = 2.0445, 21.9847, and 0, respectively; consequently, the priority order is $p_{2}, p_{1}, p_{3}$. Although the six linear regression models do not have a consistent rank on pigment types, most of them follow this order. Half of the six coefficients for pigment proportion are positive. It is thus inconclusive in determining the impact of pigment proportion on the gloss of topcoat paint.

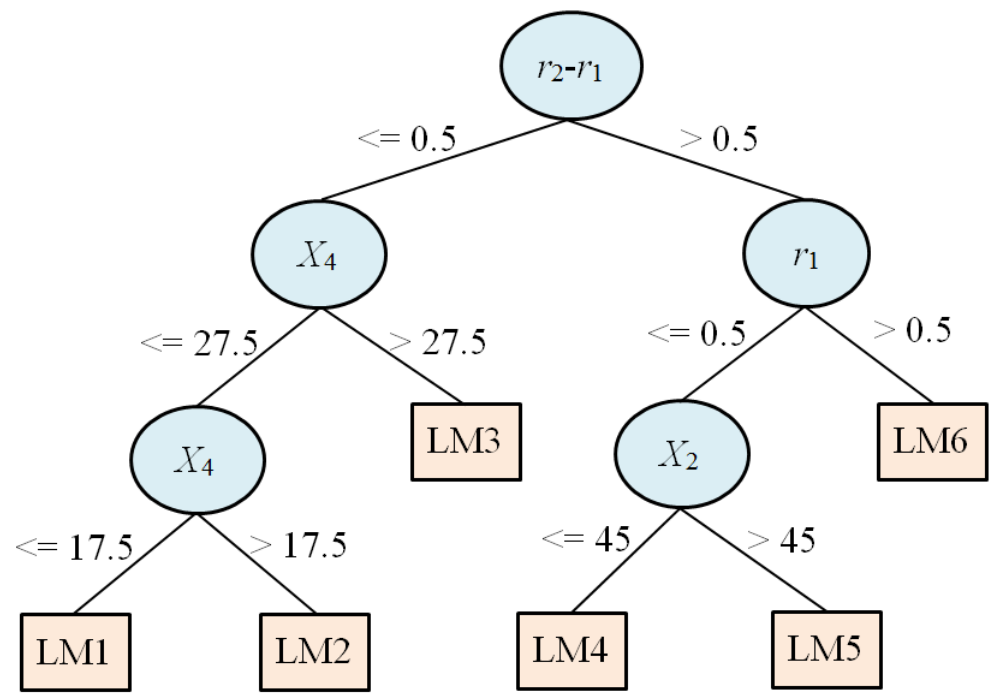

Fig. 4 The model tree grown from data set $E_{2}$

Table 7 The linear regression models for the model tree given in Fig. 4

\begin{tabular}{|c|c|c|c|c|c|c|}
\hline Attribute & LM1 & LM2 & LM3 & LM4 & LM5 & LM6 \\
\hline$r_{2}-r_{1}$ & 1.6714 & 1.6714 & 1.6714 & 0.8849 & 0.8849 & 0.8849 \\
\hline$r_{1}$ & 0.7991 & 0.7991 & 0.7991 & 1.1751 & 1.1751 & 1.1751 \\
\hline$X_{2}$ & 0.1615 & 0.3795 & 0.2530 & 0.2476 & 0.4002 & 0.2023 \\
\hline$p_{2}-p_{1}$ & 21.9847 & 2.5062 & -16.0532 & 11.8200 & -5.8016 & 6.1060 \\
\hline$p_{1}$ & -17.9402 & -7.3138 & 24.6857 & -3.3163 & 5.7759 & -4.3692 \\
\hline$X_{4}$ & -0.1888 & -1.0879 & -0.9935 & 0.3213 & 0.4718 & 0.0083 \\
\hline Constant & 58.7103 & 78.9668 & 80.5453 & 59.4366 & 44.5479 & 73.7545 \\
\hline
\end{tabular}


The above analysis is applied to all of the five model trees for the gloss retention rate, and the results are summarized in Table 8. The best and the worst resin types are $r_{1}$ and $r_{3}$, respectively. Similar to the analysis performed for the color difference, higher resin proportion is definitely beneficial for maintaining the gloss of topcoat paint. It seems that $p_{2}$ is the best among the three pigment types for the gloss retention rate. The proportion of pigment in composing topcoat paint should depend on the types of resin and pigment. Based on the results obtained from Table 8, the best formula for the gloss of topcoat paint is the combination of high proportion $r_{1}$ resin and generally low proportion $p_{2}$ pigment.

Table 8 The summarization of the linear regression models for gloss retention rate

\begin{tabular}{|c|c|c|c|c|c|}
\hline Data set & $E_{1}$ & $E_{2}$ & $E_{3}$ & $E_{4}$ & $E_{5}$ \\
\hline Rank of resin types & $r_{1}, r_{2}, r_{3}$ & $r_{1}, r_{2}, r_{3}$ & $r_{1}, r_{2}, r_{3}$ & $r_{1}, r_{2}, r_{3}$ & $r_{1}, r_{2}, r_{3}$ \\
\hline Resin proportion & Positive & Positive & Positive & Positive & Positive \\
\hline Rank if pigment types & $p_{3}, p_{1}, p_{2}$ & $p_{2}, p_{1}, p_{3}$ & $p_{2}, p_{1}, p_{3}$ & $p_{2}, p_{1}, p_{3}$ & $p_{2}, p_{3}, p_{1}$ \\
\hline Pigment proportion & Negative & Inconclusive & Inconclusive & Inconclusive & Negative \\
\hline
\end{tabular}

\section{Conclusions}

In this paper, the aging-resistance properties of topcoat paint measured by its color and gloss are investigated by numeric prediction methods. A formula of topcoat paint is mainly composed of resin and pigment. This study analyzed 180 possible formulas of resin type, resin proportion, pigment type, and pigment proportion to determine their impact on the aging-resistance properties. Ultraviolet accelerated test machines were the devices for testing the paint covered in aluminum boards to collect their color differences and gloss retention rates for every 250 hours. The data collected at the same time are gathered in a file to grow a model tree for studying the impact of the four attributes for composing topcoat paint.

The experimental results on the five data sets for the color difference consistently indicate that the best formula is the combination of a specific type of resin with a high proportion and a specific type of pigment with a low proportion. The experimental results on the five data sets for the gloss retention rate have the same conclusion on the resin type and resin proportion. However, the best pigment types for color and retention are different and the pigment proportion for gloss generally depends on the other three attributes. The information obtained in this study could be helpful in finding better formulas to satisfy the needs of customers and in choosing vendors who provide resin and pigment.

The lifetime of topcoat paint is another key factor in determining its quality. The color and gloss of paint obtained from ultraviolet accelerated test machines can be good indicators about its lifetime in natural environments. Estimating the lifetime of topcoat paint should thus be an interesting topic for the extension of this study.

\section{Acknowledgement}

This research was supported by the Ministry of Science and Technology in Taiwan under Grant No. 107-2410-H-006-045-MY3.

\section{Conflicts of Interest}

The authors declare no conflict of interest.

\section{References}

[1] A. Boubault, C. K. Ho, A. Hall, T. N. Lambert, and A. Ambrosini, "Durability of Solar Absorber Coatings and Their Cost-Effectiveness,” Solar Energy Materials and Solar Cells, vol. 166, pp. 176-184, July 2017.

[2] K. Simunkova, M. Panek, and A. Zeidler, "Comparison of Selected Properties of Shellac Varnish for Restoration and Polyurethane Varnish for Reconstruction of Historical Artefacts," Coatings, vol. 8, no. 4, March 2018.

[3] J. Wu, K. Niu, B. Su, and Y. Wang, "Effect of Combined UV Thermal and Hydrolytic Aging on Micro-Contact Properties of Silicone Elastomer,” Polymer Degradation and Stability, vol. 151, pp. 126-135, May 2018. 
[4] E. Kizilkonca and F. B. Erim, "Development of Anti-Aging and Anticorrosive Nanoceria Dispersed Alkyd Coating for Decorative and Industrial Purposes,” Coatings, vol. 9, no. 10, September 2019.

[5] P. M. Carmona-Quiroga, R. M. J. Jacobs, S. Martinez-Ramirez, and H. A. Viles, "Durability of Anti-Graffiti Coatings on Stone: Natural vs Accelerated Weathering," PLoS One, vol. 12, no. 2, February 2017.

[6] P. Sanmartin and F. Cappitelli, "Evaluation of Accelerated Ageing Tests for Metallic and Non-Metallic Graffiti Paints Applied to Stone," Coatings, vol. 7, no. 11, October 2017.

[7] A. K. Soares, R. L. Pereira, P. H. Gonzalez de Cademartori, H. W. Dalla Costa, and D. A. Gatto, "Artificial Weathering of Four Coatings Applied on Woods of Two Forest Species,” Nativa: Pesquisas Agrárias e Ambientais, vol. 6, no. 3, pp. 313-320, 2018.

[8] R. David, V. S. Raja, S. K. Singh, and P. Gore, "Development of Anti-Corrosive Paint with Improved Toughness Using Carboxyl Terminated Modified Epoxy Resin," Progress in Organic Coatings, vol. 120, pp. 58-70, July 2018.

[9] X. X. Yan, "Effect of Black Paste on the Property of Fluorine Resin/Aluminum Infrared Coating," Coatings, vol. 9, no. 10, September 2019.

[10] T. Ramde, L. G. Ecco, and S. Rossi, "Visual Appearance Durability as Function of Natural and Accelerated Ageing of Electrophoretic Styrene-Acrylic Coatings: Influence of Yellow Pigment Concentration,” Progress in Organic Coatings, vol. 103, pp. 23-32, February 2017.

[11] S. N. Roselli, R. Romagnoli, and C. Deyda, "The Anti-Corrosion Performance of Water-Borne Paints in Long Term Tests," Progress in Organic Coatings, vol. 109, pp. 172-178, August 2017.

[12] M. Kohl, A. Kalendova, E. Cernoskova, M. Blaha, J. Stejskal, and M. Erben, "Corrosion protection by organic coatings containing polyaniline salts prepared by oxidative polymerization,” Journal of Coatings Technology and Research, vol. 14, no. 6, pp. 1397-1410, November 2017.

[13] N. M. Ahmed, M. G. Mohamed, R. H. Tammam, and M. R. Mabrouk, "Performance of Coatings Containing Treated Silica Fume in the Corrosion Protection of Reinforced Concrete," Pigment \& Resin Technology, vol. 47, no. 4, pp. 350-359, July 2018.

[14] Q. M. Abd El-Gawad, N. M. Ahmed, M. M. Selim, E. Hamed, and E. R. Souaya, "The Anticorrosive Performance of Cost Saving Zeolites,” Pigment \& Resin Technology, vol. 48, no. 4, pp. 317-328, July 2019.

[15] R. S. Peres, A. V. Zmozinski, F. R. Brust, A. J. Macedo, E. Armelin, C. Aleman, and C. A. Ferreira, "Multifunctional Coatings Based on Silicone Matrix and Propolis Extract," Progress in Organic Coatings, vol. 123, pp. 223-231, October 2018.

[16] W. H. Li, D. C. Franco, M. S. Yang, X. P. Zhu, H. P. Zhang, Y. Y. Shao, H. Zhang, and J. X. Zhu, "Investigation of the Performance of ATH Powders in Organic Powder Coatings," Coatings, vol. 9, no. 2, February 2019.

[17] T. Kanbayashi, A. Ishikawa, M. Matsunaga, M. Kobayashi, and Y. Kataoka, "Application of Confocal Raman Microscopy for the Analysis of the Distribution of Wood Preservative Coatings," Coatings, vol. 9, no. 10, September 2019.

[18] A. Karalic, "Employing Linear Regression in Regression Tree Leaves," Proc. of the 10th European Conference on Artificial Intelligence, August 1992, pp. 440-441.

[19] J. R. Quinlan, "Learning with Continuous Classes," Proc. of the 5th Australian Joint Conference on Artificial Intelligence, November 1992, pp. 343-348.

[20] P. Chaudhuri, M. C. Huang, W. Y. Loh, and R. Yao, "Piecewise-Polynomial Regression Trees," Statistica Sinica, vol. 4, no. 1, pp. 143-167, January1994.

[21] W. Y. Loh, "Regression Trees with Unbiased Variable Selection and Interaction Detection," Statistica Sinica, vol. 12, no. 2, pp. 361-286, April 2002.

[22] E. Ghasemi, H. Kalhori, R. Bagherpour, and S. Yagiz, "Model Tree Approach for Predicting Uniaxial Compressive Strength and Young's Modulus of Carbonate Rocks," Bulletin of Engineering Geology and The Environment, vol. 77, no. 1, pp. 331-343, February 2018.

[23] F. T. Matthew, A. I. Adepoju, O. Ayodele, O. Olumide, O. Olatayo, E. Adebimpe, O. Bolaji, and E. Funmilola, "Development of Mobile-Interfaced Machine Learning-Based Predictive Models for Improving Students' Performance in Programming Courses," International Journal of Advanced Computer Science and Applications, vol. 9, no. 5, pp. 105-115, 2018.

[24] H. P. Su, C. Lian, J. C. Liu, and H. L. Liu, "Machine Learning Models for Solvent Effects on Electric Double Layer Capacitance,” Chemical Engineering Science, vol. 202, pp. 186-193, July 2019.

[25] H. S. Yi, B. Lee, S. Park, K. C. Kwak, and K. G. An, "Prediction of Short-Term Algal Bloom Using the M5P Model-Tree and Extreme Learning Machine," Environmental Engineering Research, vol. 24, no. 3, pp. 404-411, 2018.

[26] Z. S. Khozani, K. Khosravi, B. T. Binh, B. Klove, W. H. M. W. Mohtar, and Z. M. Yaseen, "Determination of Compound Channel Apparent Shear Stress: Application of Novel Data Mining Models,” vol. 21, no. 5, pp. 798-811, 2019. 